\title{
The Painful Hand
}

\section{Die schmerzende Hand}

\author{
Maurizio Calcagni
}

Klinik für Plastische Chirurgie und Handchirurgie, Universitätsspital Zürich, Zürich

Injuries, infections, osteoarthritis, nerve compressions, tendon inflammations of the hand and wrist are among the most commonly reported musculoskeletal problems. Many of them might look trivial and surely not threatening, but they can still have a major impact on the quality of life if poorly diagnosed or left untreated.

Carpal tunnel syndrome, trigger finger or tenosynovitis of the wrist aren't the only reasons for hand pain, there is a lot more, and even though it might sound obvious, a good treatment starts always with an accurate diagnosis. This is often challenging because of the anatomy of the hand with all the different structures packed in a small volume that can become painful, and the many different symptoms that can be misleading. This challenge, however, can be mastered with the right basic knowledge. In this issue of Praxis, we would like to present an overview of the most common reasons for hand pain and of the most often-misdiagnosed problems.

The first mini review is dedicated to the finger pathologies and specially to osteoarthritis and its treatment. This is probably the part of hand surgery that experienced the biggest evolution in the last years, thank to new joint implants that cure pain and restore function in a very reliable way. The second review summarises the most common causes of wrist pain from osteoarthritis to ligament and tendon problems, and how to differentiate between them. The third review concentrates on the tendinopathies of the hand. Most of these cases can be treated conservatively but must be carefully assessed to avoid chronification and a long-lasting function loss. The last review treats the different forms of nerve pain. This is a particularly difficult part of hand surgery, where misdiagnoses - unfortunately still quite frequent-, have an extremely high impact on the quality of life.

Hand surgery is a specialty with a big variety of treatments and rather small patient numbers for each of them, making it very difficult to gather clear evidence and to perform randomised trials. In some fields the treatments haven't changed much in the last decade, but in others a growing body of evidence allows for better indications and results. A relevant contribution to the collection of information stems from the outcome registries established over the last years, which allow us to identify the pertinent outcomes for our patients and show us the ways to improve functional results.

A Swiss hand surgery outcome registry, shared among a small group of pilot centres, has recently been established with the main goal of gathering the data necessary to refine our treatments in the interest not only of our patients, but also of all other players in health care. Based on these data, we expect soon to improve the guidelines and recommendations for the treatment of hand pain.

PD Dr. med. Maurizio Calcagni

Stv. Klinikdirektor

Klinik für Plastische Chirurgie und Handchirurgie

Universitätsspital Zürich

maurizio.calcagni@usz.ch 